\title{
- Implantação de marcapasso em cão - Relato de caso
}

- Pacemaker implantation in a dog - A case report

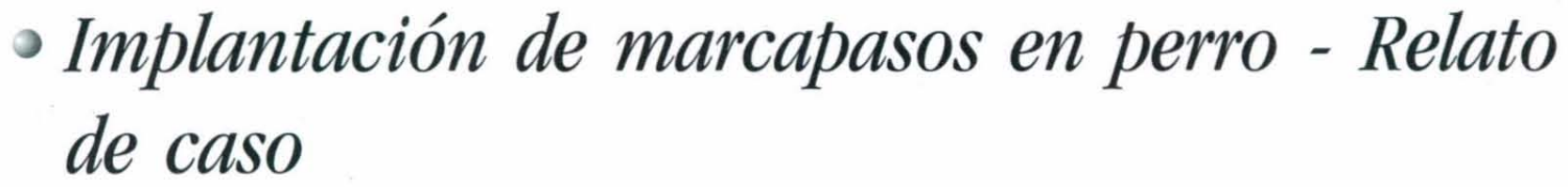

* Rodrigo Ramos de Freitas ${ }^{1}$ - CRMV-SP - $\mathbf{n}^{0} 8522$

Angelo João Stopiglia ${ }^{2}$ - CRMV-SP - $\mathbf{n}^{\circ} 1589$

Denise Tabacchi Fantoni ${ }^{3}$ - CRMV-SP $-n^{0} 5625$

Edson Azevedo Simões ${ }^{1}$ - CRMV-SP $-\mathrm{n}^{0} 8484$

Maria Helena Matiko Akao Larsson ${ }^{4}$ - CRMV-SP - no 0796

Fábio Biscegli Jatene ${ }^{5}$ - CRM-SP - no 33865

Luciana Oliveira Domingos Barbusci ${ }^{6}$ - CRMV-SP $-n^{0} 8493$

Elisângela Aneli ${ }^{7}$

Eduardo Toshio Irino ${ }^{7}$

1 Pós-graduando em Cirurgia do Departamento de Cirurgia - Laboratório de Cirurgia Cardiotorácica - FMVZ - USP/SP.

Professor Titular do Departamento de Cirurgia da FMVZ-USP/SP.

3 Professor Associado do Departamento de Cirurgia da FMVZ-USP/SP.

4 Professor Associado do Departamento de Clínica Médica da FMVZ-USP/SP.

5 Professor Associado do Departamento de Cirurgia da FM-USP/SP.

6 Pós-graduanda em Anatomia do Departamento de Cirurgia da FMVZ-USP/SP.

Discentes de Medicina Veterinária da FMVZ-USP/SP.

\section{RESUMO}

Um animal da espécie canina, fềmea, raça Maltês, com um ano e seis meses de idade e pesando 3,2 quilogramas apresentava histórico de pré-síncope, sopro em foco mitral e bradicardia pela auscultação. Submeteu-se o animal a exame eletrocardiográfico e radiográfico, diagnosticando-se bloqueio atrioventricular de $3^{\circ}$ grau e o tratamento recomendado foi a implantação de marcapasso. O período pós-operatório transcorreu normalmente e o animal apresenta bom estado geral, não apresentando nenhuma alteração clínica até o presente momento.

Palavras-chave: Bloqueio atrioventricular. Marcapasso. Cão. 


\section{Introdução}

$\underline{0}$ implante de marcapasso artificial em cães vem se tornando técnica muito difundida em países como os Estados Unidos e Alemanha para o tratamento de determinados distúrbios do ritmo cardíaco como, por exemplo, o bloqueio atrioventricular de terceiro grau. Em 1968, foi realizado o primeiro caso de implante de marcapasso bem sucedido num cão e, desde então, tal intervenção cirúrgica vem sendo relatada com sucesso nesta espécie (LONG, 1989; COBB; NOLAN, 1990).

Processos patológicos adquiridos ou congênitos envolvendo o sistema de geração e condução dos estímulos (principalmente no nodo $\mathrm{A}-\mathrm{V}$ ) podem originar o bloqueio atrioventricular (BRAUNWALD, 1997) levando a bradicardia por retardar parcialmente ou impedir completamente que as despolarizações originadas no nodo S-A prossigam para os ventrículos (BUCHANAN; DEAR; PYLE, 1968; ETTINGER, 1992).

Traumatismos, crescimentos invasivos do miocárdio, defeitos congênitos do coração, dilatação atrial, fibroses do tecido juncional atrioventricular, intoxicação por digitálicos e dirofilariose podem estar relacionados com o desenvolvimento de tal bloqueio (ETTINGER, 1992; FINGEROTH, 1994).

Os bloqueios atrioventriculares são classificados em três graus. Nos bloqueios de primeiro grau não há necessidade de tratamento e raramente se detecta a doença, pois o animal não apresenta sinais clínicos; já, nos de segundo grau, principalmente em estágios mais avançados, é necessária em alguns casos até mesmo a implantação de marcapasso artificial (ETTINGER, 1992; FINGEROTH, 1994). O bloqueio atrioventricular de terceiro grau, também denominado completo ou total, é caracterizado por não permitir que nenhum impulso proveniente do nodo $\mathrm{S}-\mathrm{A}$ ou nodo $\mathrm{A}-\mathrm{V}$ passe para os ventrículos (BLACK; BRADLEY, 1983; ETTINGER, 1992; BRAUNWALD, 1997), causando intensa bradicardia, que pode levar os animais à intolerância a exercícios físicos, apatia, pré-síncope, síncope e insuficiência cardíaca congestiva (BUCHANAN; DEAR; PYLE, 1968; ETTINGER, 1992; BRAUNWALD, 1997). Os batimentos ventriculares, dependendo da raça, são inferiores a 40 por minuto (DARKE; BEEN; MARKS, 1985), ocorrendo, no entanto, em ritmo regular (BUCHANAN; DEAR; PYLE, 1968; KIRKLIN; BARRAT-BOYES, 1993).

O eletrocardiograma, principal exame no diagnóstico desta doença, mostra ondas $\mathrm{P}$ em maior freqüência, independentes dos complexos QRS
(BUCHANAN; DEAR; PYLE, 1968; BLACK; BRADLEY, 1983; FINGEROTH, 1994). Exames radiológicos e ecocardiográficos podem auxiliar no diagnóstico do bloqueio atrioventricular total (SISSON et al., 1991). Este distúrbio tende a ser irreversível e a recomendação terapêutica é a implantação de marcapasso (LONG, 1989; COBB; NOLAN, 1990; SISSON et al., 1991; ETTINGER, 1992; FINGEROTH, 1994).

Os marcapassos são classificados por meio de sigla, basicamente formada por três letras, que indicam o seu modo de funcionamento (CHARDACK, 1960; LOMBARD; TILLEY; YOSHIOKA, 1981). A primeira letra mostra qual a região do coração está sendo estimulada pelo eletrodo: átrios (A), ventrículos (V), ambos (D) ou nenhum (O). A segunda letra mostra a região que, sensibilizada pelo marcapasso, responde ao gerador sobre o estímulo sofrido, confirmando o local de estimulação: A, V, D ou O, assim como a primeira classificação. Já a terceira letra fornece o modo de resposta do aparelho ante às regiões sensibilizadas: inibição (I), inibe o gerador de estímulos, caso os batimentos ultrapassem o limite pré-determinado pelo aparelho; o gatilho para a estimulação do gerador (T) ou ainda ambas as respostas (D) (CHARDACK, 1960; LOMBARD; TILLEY; YOSHIOKA, 1981; BLACK; BRADLEY, 1983; FINGEROTH, 1994).

Atualmente há duas técnicas para o implante nos animais (BLACK; BRADLEY, 1983; SISSON et al., 1991; FINGEROTH, 1994). A primeira delas é realiza pela via endovenosa, em que a extremidade do eletrodo chega ao coração por meio da veia jugular, com o auxílio do fluoroscópio (SISSON et al., 1991). A segunda técnica é denominada epicárdica, pois diferentemente do procedimento anterior, o eletrodo é conectado ao epicárdio e não ao endocárdio. Na técnica epicárdica, após toracotomia intercostal, uma das extremidades do eletrodo é alojada na parede cardíaca, enquanto a outra extremidade transpassa o diafragma e se conecta ao gerador de pulsos, que pode ser fixado tanto no espaço subcutâneo, parede abdominal ou permanecer livre na cavidade abdominal (DARKE; BEEN; MARKS, 1985; COBB; NOLAN, 1990; SISSON et al., 1991; ETTINGER, 1992).

Complicações como migração do gerador, estimulação muscular local, formação de seroma e desalojamento do eletrodo podem acontecer em decorrência do mau posicionamento do gerador de pulsos e do cabo-eletrodo (BUCHANAN; DEAR; PYLE, 1968; LOMBARD; TYLLEY; YOSHIOKA, 1981; YOSHIOKA et al., 1981; BONAGURA; HELPHERY; MUIR, 1983). 
Já foi relatado caso de formação neoplásica ao redor do gerador implantado na região subcutânea, identificado como mixoma, porém exames realizados no parênquima tumoral e na cápsula do gerador não revelaram possíveis depósitos de metais potencialmente carcinogênicos (ROWLAND; MOISE; SEVERSON, 1991).

\section{Relato do caso}

No dia 20/04/99, um animal da espécie canina, fềmea, raça Maltês, com um ano e seis meses de idade e pesando 3,2 quilogramas, foi encaminhado ao Serviço de Cardiologia do Hospital Veterinário da Faculdade de Medicina Veterinária e Zootecnia da Universidade de São Paulo. O proprietário relatou episódios de pré-síncope e, pela auscultação, constatou-se sopro sistólico em foco mitral e bradicardia. Ao exame físico, o animal apresentou bom estado geral e os outros sistemas orgânicos não apresentavam alterações dignas de nota.

Pelo eletrocardiograma, verificou-se freqüência ventricular variável, tendo como média aproximadamente $40 \mathrm{bpm}$ e onda $\mathrm{P}$ com 0,04s x 0,3-0,6 mV (Figura 1). Pela análise radiográfica, observou-se acentuada cardiomegalia e discreta densificação de campos pulmonares. Foi ainda realizado exame para dirofilariose, cujo resultado foi negativo.

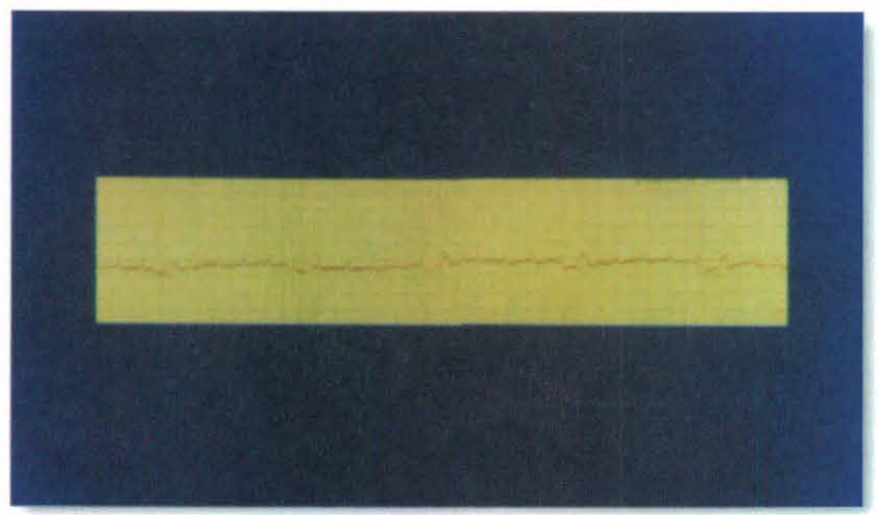

Figura 1 - Eletrocardiograma (D2-25mm/s); pré-implantação do marcapasso artificial: bloqueio atrioventricular completo do nodo atrioventricular; ondas P não são seguidas de batimentos ou deflexos do Feixe de His, porém cada complexo QRS é precedido por deflexão do Feixe de His.

Diagnosticou-se bloqueio atrioventricular de $3^{\circ}$ grau, e o tratamento recomendado foi a implantação de marcapasso. Nenhum medicamento foi prescrito.

A avaliação pré-operatória consistiu-se de exame de função renal, hepática, hemograma e urinálise, não sendo detectada qualquer anormalidade. Nove dias depois, o animal foi encaminhado ao Laboratório de Cirurgia Cardiotorácica, da mesma instituição, para a cirurgia de implante de marcapasso.

Como medicação pré-anestésica, foi administrado, pela sua ação vagolítica, cloridrato de meperidina (Dolosal - Cristália - Brasil) na dose de $2 \mathrm{mg} / \mathrm{kg}$ pela via intramuscular. Decorridos quinze minutos, a indução foi obtida com midazolam (Dormonid - Roche - Brasil) na dose de $0,5 \mathrm{mg} / \mathrm{kg}$ e cloridrato de quetamina (Ketalar Aché - Brasil) na dose de $0,5 \mathrm{mg} / \mathrm{kg}$ via endovenosa. A manutenção da anestesia foi mantida com isofluorano (Isoflurone - Cristália - Brasil). Durante o procedimento cirúrgico, a monitorização do paciente consistiu em traçado eletrocardiográfico, pressão arterial por método invasivo, capnografia, oximetria de pulso, gases anestésicos no ar expirado e inspirado e $\mathrm{FiO}_{2}$.

$\mathrm{O}$ acesso ao coração foi feito através da toracotomia lateral esquerda, no quinto espaço intercostal. O cabo-eletrodo foi fixado no epicárdio do ventrículo esquerdo em região menos vascularizada (Figura 2), passado pelo diafragma e conectado ao gerador de pulsos, alojado na região subcutânea do flanco esquerdo.

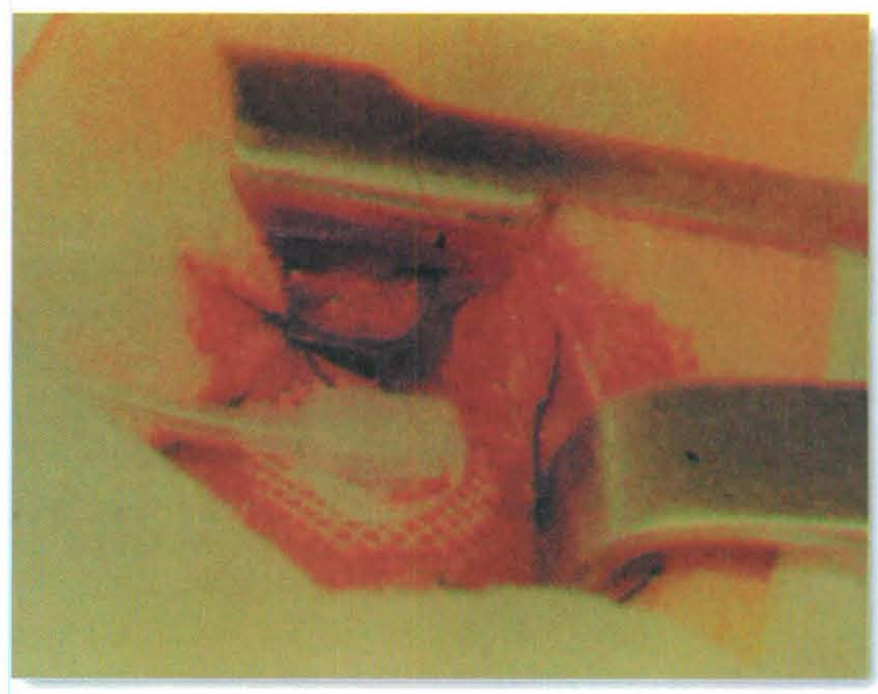

Figura 2 - Eletrocardiograma (D2-25mm/s); pós-implantação do marcapasso artificial: eletrodo implantado em ventrículo esquerdo padrão eletrocardiográfico de bloqueio de ramo direito

O modelo do gerador de pulsos utilizado na paciente foi o Eikos SLD da marca Biotronik (Figura 3), com durabilidade média de 6 a 7 anos, com programação de fábrica VDD, embora no momento da implantação foi configurado para atuar como VVI por ser o arranjo mais adequado à paciente, já que o bloqueio atrioventricular de $3^{\circ}$ grau leva aà bradicardia ventricular, necessitando, assim, de um marcapasso estimulando esta região do coração $(\mathrm{V})$, recebendo a resposta da região 
do coração estimulada (V) e inibindo a ação do gerador de pulsos, caso os batimentos cardíacos ultrapassassem $84 \mathrm{bpm}(\mathrm{I})^{12}$. Já o cabo-eletrodo utilizado foi o 5071 da marca Medtronic - tipo "screw-in", conhecido no Brasil como tipo "parafuso" ou "saca-rolha".

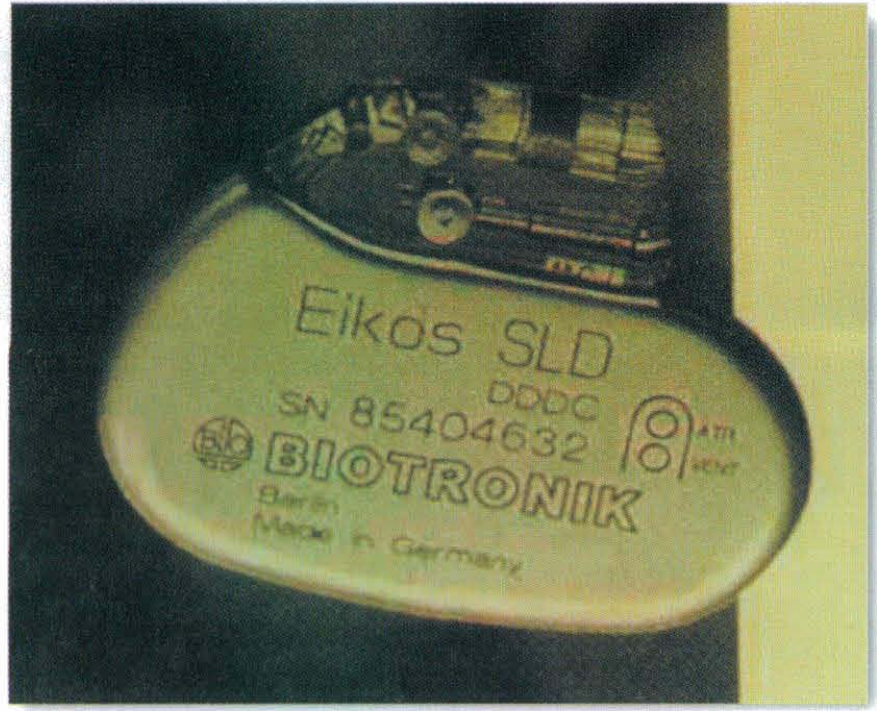

Figura 3 - Implantação do eletrodo em ventrículo esquerdo.

Um dreno torácico do tipo selo d'água (Física Incor - Brasil) foi colocado na cavidade torácica, após fechamento do tórax e permanecendo por três horas, para permitir o restabelecimento de pressão negativa necessária dentro desta cavidade. Ao término do procedimento, administraram-se duas doses de cloridrato de morfina (Dimorf - Cristália - Brasil) na dose de $0,5 \mathrm{mg} / \mathrm{kg}$, pela via intramuscular, com intervalo de quatro horas para a obtenção de analgesia e prescreveu-se ainda cefalexina (Keflex - Eli Lilly - Brasil) na dose de $15 \mathrm{mg} / \mathrm{kg}$, pela via oral, por sete dias.

Após sete dias, foram retirados os pontos cirúrgicos e realizado raio $\mathrm{X}$ de tórax (Figura 4), observando-se ainda o mesmo aumento global da silhueta cardíaca, com deslocamento dorsal da traquéia, pois ainda não houve tempo suficiente para o coração retornar à sua silhueta normal. Não foi encontrada nenhuma alteração radiográfica em campos pulmonares. O ECG apresentou o traçado esperado pós-implantação (Figura 5), com a freqüência determinada pelo marcapasso estável em 84 bpm.

Em razão do pequeno porte do animal e da incapacidade da pele em apresentar elasticidade suficiente para comportar o tamanho do gerador de pulsos na região do flanco, ocorreu o rompimento da pele e exposição do flanco. No dia $03 / 09 / 99$, foi realizada nova intervenção cirúrgica para colocação do marcapasso dentro da cavidade abdominal, sendo fixado à sua mus- culatura, na região ventral (Figura 6). Após sete dias, os pontos cirúrgicos foram retirados, não se alterando, em nenhum momento, o bom estado clínico que o animal apresentava desde a cirurgia de implantação de marcapasso.

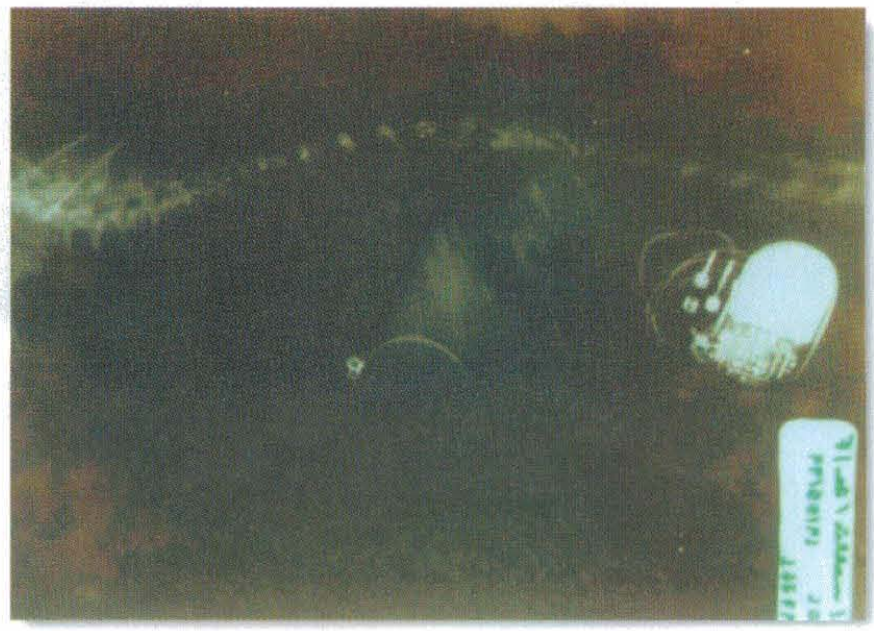

Figura 4 - Gerador de pulsos Eikos SLD, da Biotronik

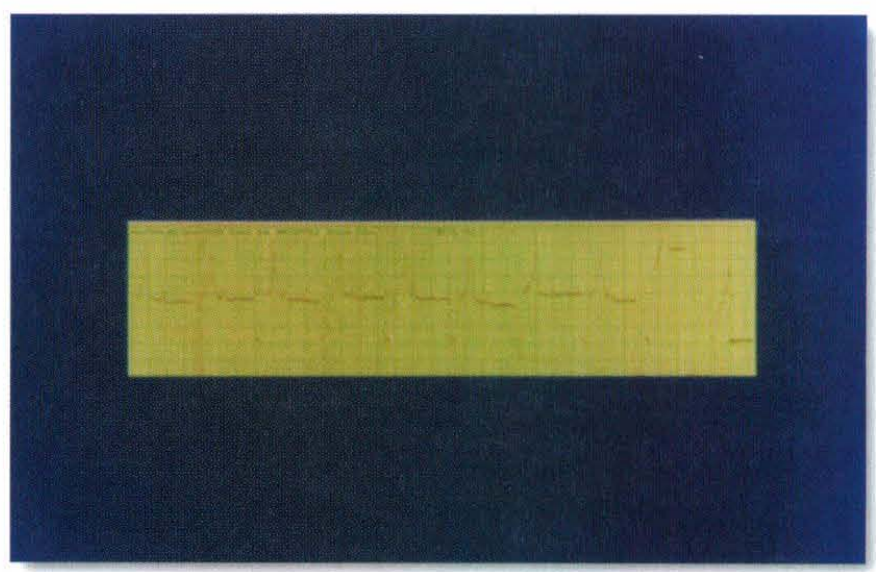

Figura 5 - Radiografia do tórax em posição látero-lateral; pós-implantação do gerador de marcapasso no subcutâneo do flanco esquerdo. Nota-se bem o gerador de impulsos (marcapasso), o cabo eletrodo e o eletrodo tipo "parafuso" ou "saca-rolha", implantado no epicárdio (ventrículo esquerdo).

O período pós-operatório transcorreu normalmente, como na primeira intervenção cirúrgica. $\mathrm{O}$ animal apresenta bom estado geral e nenhuma alteração clínica foi notada até o presente momento. Foi recomendado ao proprietário o retorno anual do animal ao Hospital Veterinário para avaliação clínica e verificação do funcionamento do marcapasso e desgaste da bateria.

O aparelho está programado para fornecer freqüência de 84 bpm, e como a duração média da bateria com essa programação é de 6-7 anos, o animal poderá vir a sofrer mais uma intervenção para ser trocada. O paciente necessitaria, pela sua fisiologia, de 


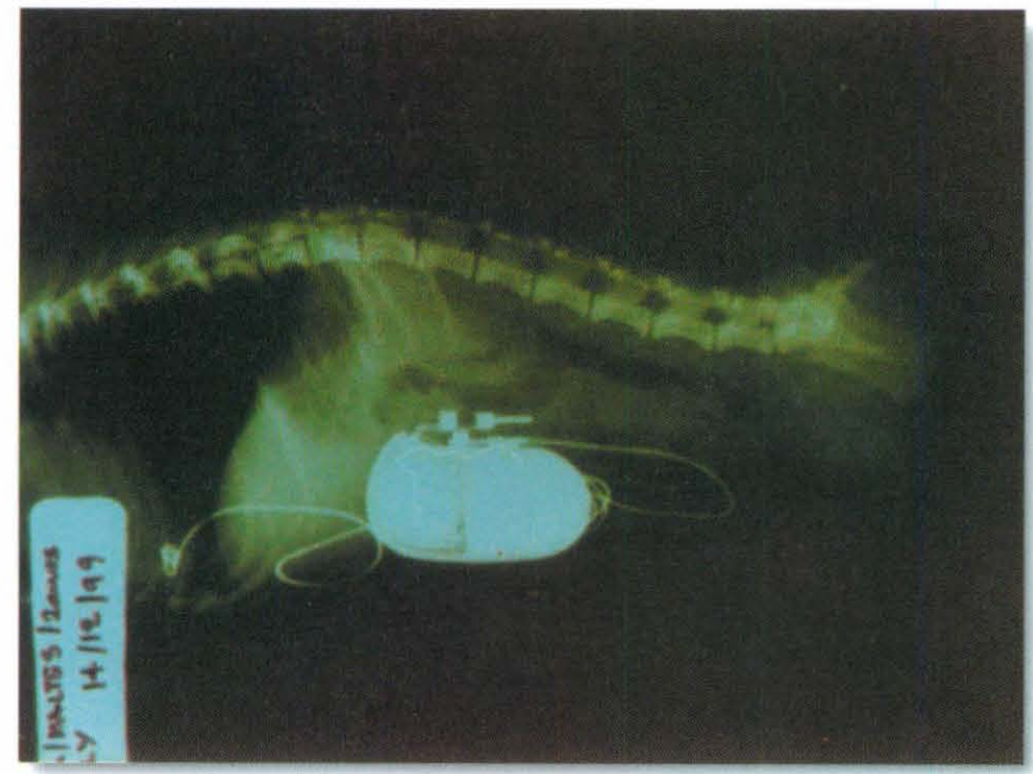

Figura 6 - Radiografia do tórax em posição látero-lateral, mostrando gerador de marcapasso implantado na cavidade abdominal, após nova intervenção. São visualizados o gerador de impulsos (marcapasso), o cabo eletrodo e o eletrodo tipo "parafuso" ou "saca-rolha", implantado no epicárdio (ventrículo esquerdo).

uma freqüência cardíaca maior, mas por uma limitação do aparelho de gerador de pulsos, que possuía como freqüência máxima $85 \mathrm{bpm}$, houvemos por bem instituir a freqüência de $84 \mathrm{bpm}$. O paciente não apresentou nenhum sintoma clínico de insuficiência cardíaca pósimplantação, fazendo-nos crer que a freqüência escolhida está satisfazendo as necessidades fisiológicas do seu organismo.

\section{Discussão}

Segundo a literatura consultada, a implantação de marcapasso é o tratamento de escolha para cães com bloqueio atrioventricular de $2^{\circ} \mathrm{e} 3^{\circ}$ grau (BONAGURA; HELPHERY; MUIR, 1983; BRAUNWALD, 1997), exceto quando causado por intoxicação severa com digitálicos (LOMBARD; TILLEY; YOSHIOKA, 1981). Corroborando esta mesma idéia, está o fato de que a implantação de marcapasso vem sendo utilizada desde a década de 60 em humanos (BUCHANAN; DEAR; PYCE, 1968) e, atualmente, é procedimento rotineiro nos hospitais veterinários que possuem o serviço de implantação de marcapasso.

Pré-síncope, síncope, apatia, intolerância a exercícios e insuficiência cardíaca congestiva são sinais freqüentes em animais com este distúrbio de condução (BRAUNWALD, 1997; ETTINGER, 1992; FINGEROTH, 1994). Atropina e isoproterenol podem ser usados na terapia médica (BUCHANAN; DEAR;
PYCE, 1968; LOMBARD; TILLEY; YOSHIOKA, 1981; BRAUNWALD, 1997), mas não podem aumentar ou manter a freqüência cardíaca em níveis desejáveis por várias horas ou dias em pacientes sintomáticos (BRAUNWALD, 1997). No caso descrito, o paciente não recebeu nenhum medicamento, optando-se pela rápida intervenção cirúrgica antes que o quadro geral do paciente se deteriorasse.

No homem, o ECG pode exibir dois traçados, dependendo da região do sistema de condução em que está localizado o bloqueio (BRAUNWALD, 1997). Focos próximos ao feixe de His geralmente exibem complexo QRS normal, pois o foco ectópico que controla o ventrículo está no feixe de His ou próximo a ele, definindo tal traçado. Já lesões no nodo $\mathrm{A}-\mathrm{V}$ apresentam cada complexo ventricular precedido por uma deflexão do feixe de His (BRAUNWALD, 1997). O animal apresentava o segundo tipo de traçado, o que sugere que este possuía uma lesão no nodo AV.

A toracotomia lateral esquerda foi a técnica cirúrgica escolhida para se acessar o coração. Através do $5^{\circ}$ espaço intercostal, pode-se visualizar o ápice do ventrículo esquerdo. O eletrodo foi implantado em região pouco vascularizada, evitando-se o risco de hemorragias severas. Preferiu-se esta técnica (epicárdica) ao acesso endovenoso (endocárdico) pois o posicionamento e fixação do eletrodo são mais confiáveis desta forma do que no método transvenoso, no qual movimentos da cabeça ocasionalmente causam deslocamento do eletrodo, causando falhas no funcionamento do marcapasso (BUCHANAN; DEAR; PYCE, 1968). Outro fator limitante foi a indisponibilidade da utilização de um fluoroscópio, aparelho imprescindível na fixação endocárdica, pela técnica endovenosa (SISSON et al., 1991). O eletrodo em formato de "rosca", usado nesta cirurgia, possibilita rápida e fácil implantação. Uma outra opção cirúrgica seria a técnica transdiafragmática, relatada como sendo um procedimento cirúrgico que requer menos tempo de execução e ocasiona menor dor pós-operatória (FOX et al., 1986). No caso relatado, preferimos a toracotomia intercostal esquerda por permitir uma melhor visualização do ventrículo esquerdo e pela equipe cirúrgica estar melhor adaptada a esta técnica. No caso em apreço, o paciente não apresentou sinais de dor e as doses administradas de morfina mostraram-se suficientes para manter o paciente em analgesia nas primeiras horas após a cirurgia. 
O gerador de pulsos foi alojado no flanco esquerdo, no tecido subcutâneo. Esta técnica apresentou complicações devidas ao pequeno porte da raça do paciente e a impossibilidade de a pele apresentar elasticidade suficiente para suportar o gerador, rompendo e expondo-o, conforme relatam alguns autores (LOMBARD; TILLEY; YOSHIOKA, 1981; YOSHIOKA et al., 1981; BONAGURA; HELPHERY; MUIR, 1983). Foi novamente implantado, desta vez na cavidade abdominal, fixado ao peritônio e à musculatura adjacente na região ventral da cavidade. A disposição do gerador de pulsos dentro da cavidade abdominal foi escolhida por ser uma técnica alternativa que não costuma apresentar complicações, se comparada à implantação no flanco (BONAGURA; HELPHERY; MUIR, 1983; FOX et al., 1986).

O gerador de pulsos utilizado na paciente foi programado para atuar como VVI, apresentando como vantagem uma maior durabilidade (KIRKLIN; BARRAT-BOYES, 1993).

\section{SUMMARY}

A female Maltese dog, 18 months old, with $3.2 \mathrm{~kg}$, was presented with a pre-syncope history, auscultation revealing murmur over the mitral valve area bradycardia. The animal was submitted to electrocardiography and radiographic exams, the diagnosis being third-degree atrioventricular blockade. The recommended treatment was pacemaker implantation. Postoperative evolution was normal and the animal was in good physical condition. No clinical alterations have been detected since the implantation.

Key words: Atrioventricular blockade. Pacemaker. Dog.

\section{RESUMEN}

Un animal de la especie canina, hembra, raza Maltés, con un año y seis meses de edad y pesando 3,2 kilogramos presentaba una historia clínica de pre-síncope, soplo en el foco mitral y bradicardia ante la auscultación. Se sometió al animal a un examen electrocardiográfico y radiográfico, diagnosticándose bloqueo auriculoventricular de $3^{\circ}$ grado y el tratamiento recomendado fue la implantación de un marcapasos. El período postoperatorio transcurrió normalmente y el animal presenta buen estado general, no presentando ninguna alteración clínica hasta el momento.

Palabras clave: Bloqueo auriculoventricular. Marcapaso. Perro.

\section{REFERÊNCIAS}

BLACK, A. P.; BRADLEY, W. A. Implantation of a permanent pacemaker in a dog with third degree heart block. Australian Veterinary Practitioner, v. 13, n. 3, p. 122-124, 1983.

BONAGURA, J. D.; HELPHERY, M. L.; MUIR, W. W. Complications associated with permanent pacemaker implantation in the dog. Journal of the American Veterinary Medical Association, v. 182, n. 2, p. 149-155, 1983.
BRAUNWALD, E. Heart disease. 5. ed. New York: Churchill Livingstone, $1997.535 \mathrm{p}$.

BUCHANAN, J. W.; DEAR, M. G.; PYLE, R. L. Medical and pacemaker therapy of complete heart block and congestive heart failure in a dog. Journal of the American Veterinary Medical Association, v. 152, n. 8, p. 1099-1109, 1968. 
CHARDACK, W. M. A transistorized self-contained implantable pacemaker for the long-term correction of complete heart block. Surgery, v. 48, p. 643, 1960.

COBB, M. A.; NOLAN, J. Use of a programmable, activitysensing, rate-regulating pacemaker in a dog. Journal of Small Animal Practice, v. 31, n. 8, p. 398-400, 1990.

DARKE, P. G. G.; BEEN, M.; MARKS, A. Use a programmable, "physiological" cardiac pacemaker in a dog with total atrioventricular block (with some comments and complications associated with cardiac pacemakers). Journal of Small Animal Practice, v. 26, n. 6, p. 295-303, 1985.

ETTINGER, S. J. Bloqueio atrioventricular. In: Tratado de medicina interna veterinária. 3 . ed. Philadelphia: W.B. Saunders, 1992. v. 2, p. 1143-1150.

FINGEROTH, J. M. Pacemaker teraphy for bradycardias. Seminars in Veterinary Medicine and Surgery (Small Animal), v. 9, n. 4, p. 192-199, 1994.

FOX, P. R. et al. Ventral abdominal transdiaphragmatic approach for implantation of cardiac pacemakers in the dog. Journal of the American Veterinary Medical Association, v. 189, n. 10, p. 1303-1308, 1986.
KIRKLIN, J. W.; BARRAT-BOYES, B. G. Cardiac rhythm disturbance. In: _. Cardiac surgery. $2^{\text {nd }}$ ed. New York: Churchill Livingstone, 1993. v. 2, p. 1599-1610.

LOMBARD, C. W.; TILLEY, L. P.; YOSHIOKA, M. M. Pacemaker implantation in the dog: survey and literature review. Journal of the American Animal Hospital Association, v. 17, p. $751-758,1981$.

LONG, R. D. Technique for pacemaker placement in private practice: a case report. Veterinary Record, p. 353, 1989.

ROWLAND, P. H.; MOISE, N. S.; SEVERSON, D. Myxoma at the site of a subcutanerous pacemaker in a dog. Journal of the American Animal Hospital Association, v. 27, n. 6, p. 649-651, 1991.

SISSON, P. et al. Permanent transvenous pacemaker implantation in forty dogs. Journal of Veterinary Internal Medicine, $v$. 5, n. 6, p. 322-331, 1991.

YOSHIOKA, M. M. et al. Permanent pacemaker implantation in the dog. Journal of the American Animal Hospital Association, v. 17, p. 746-750, 1981. 